\title{
LAGRANGIAN SURFACES OF CONSTANT CURVATURE IN COMPLEX EUCLIDEAN PLANE
}

\author{
BANG-YEN CHEN
}

(Received November 6, 2002, revised December 15, 2003)

\begin{abstract}
In this article we completely classify Lagrangian $H$-umbilical surfaces of constant curvature in complex Euclidean plane.
\end{abstract}

1. Lagrangian $H$-umbilical surfaces with $\lambda=2 \mu$. We follow the notation and definitions given in $[1,2]$. Professors I. Castro and F. Urbano kindly pointed out that Theorem 3.1 in [1] holds only for submanifolds of dimension $\geq 3$.

For $n=2$, we have the following result instead.

THEOREM 1. The following two statements hold:

(i) Let $b$ be a nonzero number, $\theta(t)$ a function of one variable defined on an open interval $(\alpha, \beta) \ni 0$ and $z(t)$ a $C^{2}$-valued solution of the ordinary differential equation:

$$
z^{\prime \prime}(t)-i \theta^{\prime}(t) z^{\prime}(t)+b^{2} z(t)=0,
$$

satisfying the two conditions: $|z(t)|^{2}=1 /\left(4 b^{2}\right)$ and $\left|z^{\prime}(t)\right|^{2}=1 / 4$. Then the map

$$
L(s, t)=e^{2 i b s} z(t)+\int_{0}^{t} z^{\prime}(t) e^{-2 i \theta(t)} d t
$$

defines a Lagrangian $H$-umbilical surface of constant curvature $b^{2}$ in $C^{2}$ whose induced metric is

$$
g=d s^{2}+\cos ^{2}(b s+\theta(t)) d t^{2},
$$

and whose second fundamental form satisfies

$$
h\left(e_{1}, e_{1}\right)=2 b J e_{1}, \quad h\left(e_{1}, e_{2}\right)=b J e_{2}, \quad h\left(e_{2}, e_{2}\right)=b J e_{1}
$$

with respect to $e_{1}=\partial / \partial s$ and $e_{2}=\sec (b s+\theta(t)) \partial / \partial t$.

(ii) Conversely, if $\tilde{L}: M \rightarrow C^{2}$ is a Lagrangian $H$-umbilical surface whose second fundamental form satisfies (1.4) for some function $b \neq 0$ with respect to some orthonormal local frame field $\left\{e_{1}, e_{2}\right\}$, then we have:

(ii-1) $b$ is a constant,

(ii-2) there exist a function $\theta(t)$ and a local coordinate system $\{s, t\}$ on $M$ with $e_{1}=$ $\partial / \partial$ s such that the metric tensor is given by (1.3), and

2000 Mathematics Subject Classification. Primary 53D12; Secondary 53C40, 53C42.

Key words and phrases. Lagrangian surface, Lagrangian $H$-umbilical surface, Legendre curve, surface of constant curvature, complex extensor. 
(ii-3) the immersion $\tilde{L}$ is congruent to the L given in statement (i).

PROOF. Let $b$ be a nonzero number, $\theta(t)$ a function of one variable defined on $(\alpha, \beta) \ni$ 0 and $z$ a $C^{2}$-valued solution of (1.1) satisfying $|z|^{2}=1 /\left(4 b^{2}\right)$ and $\left|z^{\prime}\right|^{2}=1 / 4$. Define a map $L$ by (1.2). Then we have

$$
\begin{aligned}
& L_{s}=2 i b e^{2 i b s} z(t), \quad L_{t}=\left(e^{2 i b s}+e^{-2 i \theta}\right) z^{\prime}(t), \\
& L_{s s}=-4 b^{2} e^{2 i b s} z(t), \quad L_{s t}=2 i b e^{2 i b s} z^{\prime}(t), \\
& L_{t t}=\left(e^{2 i b s}+e^{-2 i \theta}\right) z^{\prime \prime}(t)-2 i e^{-2 i \theta} \theta^{\prime}(t) z^{\prime}(t) .
\end{aligned}
$$

It follows from $|z|^{2}=1 /\left(4 b^{2}\right),\left|z^{\prime}\right|^{2}=1 / 4$ and (1.5) that the metric induced by $L$ is given by (1.3). Moreover, a direct computation shows that $L$ satisfies

$$
\begin{aligned}
& L_{s s}=2 i b L_{s}, \\
& L_{s t}=i b \sec (b s+\theta) e^{i(b s+\theta)} L_{t}, \\
& L_{t t}=i b \cos (b s+\theta) e^{-i(b s+\theta)} L_{s}-\theta^{\prime} \tan (b s+\theta) L_{t},
\end{aligned}
$$

by virtue of (1.1). Thus, $L$ is a Lagrangian $H$-umbilical immersion whose second fundamental form satisfies (1.4) with respect to $e_{1}=\partial / \partial s, e_{2}=\sec (b s+\theta(t)) \partial / \partial t$.

Conversely, assume that $\tilde{L}: M \rightarrow C^{2}$ is a Lagrangian $H$-umbilical immersion satisfying (1.4) for some function $b \neq 0$ with respect to some orthonormal frame field. Then (1.4) and the equation of Codazzi imply that $b$ is constant and the integral curves of $e_{1}$ are geodesic in $M$. Thus, there exists a local coordinate system $\{s, u\}$ with $e_{1}=\partial / \partial s$ so that the metric tensor of $M$ is $g=d s^{2}+f^{2}(s, u) d u^{2}$. Since the Gaussian curvature $K$ of $M$ satisfies $K=-f_{s s} / f$, (1.4) implies that $f_{s s}=-b^{2} f$. Solving this equation yields $f=A(u) \cos (b s)+B(u) \sin (b s)$ for some functions $A(u)$ and $B(u)$. Thus we get

$$
g=d s^{2}+r^{2}(u) \cos ^{2}(b s+\varphi(u)) d u^{2},
$$

where $A=r(u) \cos \varphi(u), B=r(u) \sin \varphi(u)$ and $r(u)=\sqrt{A^{2}+B^{2}}$. If we denote by $t=$ $t(u)$ an antiderivative of $r(u)$, then (1.7) becomes

$$
g=d s^{2}+\cos ^{2}(b s+\theta(t)) d t^{2}
$$

for some function $\theta(t)$. From (1.8) we find

$$
\begin{aligned}
& \nabla_{\partial / \partial s} \frac{\partial}{\partial s}=0, \quad \nabla_{\partial / \partial s} \frac{\partial}{\partial t}=-b \tan (b s+\theta(t)) \frac{\partial}{\partial t}, \\
& \nabla_{\partial / \partial t} \frac{\partial}{\partial t}=\frac{b}{2} \sin (2 b s+2 \theta(t)) \frac{\partial}{\partial s}-\theta^{\prime}(t) \tan (b s+\theta(t)) \frac{\partial}{\partial t} .
\end{aligned}
$$

Using (1.4), (1.8), (1.9) and the formula of Gauss, we see that the immersion satisfies the following system of PDEs:

$$
\begin{aligned}
& \tilde{L}_{s s}=2 i b \tilde{L}_{s}, \\
& \tilde{L}_{s t}=i b \sec (b s+\theta) e^{i(b s+\theta)} \tilde{L}_{t}, \\
& \tilde{L}_{t t}=i b \cos (b s+\theta) e^{-i(b s+\theta)} \tilde{L}_{s}-\theta^{\prime} \tan (b s+\theta) \tilde{L}_{t} .
\end{aligned}
$$


After solving the first and the second equations in (1.10), we obtain

$$
\tilde{L}=A(t) e^{2 i b s}+B(t)
$$

for some $C^{2}$-valued functions $A(t), B(t)$. Differentiating (1.11) gives

$$
\tilde{L}_{t}=A^{\prime}(t) e^{2 i b s}+B^{\prime}(t) .
$$

Substituting this into the second equation in (1.10) gives

$$
B^{\prime}(t)=e^{-2 i \theta} A^{\prime}(t) \text {. }
$$

Combining (1.12) and (1.13) yields

$$
\tilde{L}_{t}=A^{\prime}(t)\left(e^{2 i b s}+e^{-2 i \theta}\right) \text {. }
$$

Thus, after applying a suitable translation, we discover that

$$
\tilde{L}=(A(t)+C) e^{2 i b s}+\int_{0}^{t} A^{\prime}(t) e^{-2 i \theta(t)} d t
$$

for some constant vector $C$. Therefore, if we put $z(t)=A(t)+C$, we obtain (1.2).

Using (1.2), we find

$$
\tilde{L}_{s}=2 i b e^{2 i b s} z(t), \quad \tilde{L}_{t}=\left(e^{2 i b s}+e^{-2 i \theta}\right) z^{\prime}(t),
$$

which implies that $\left|L_{s}\right|^{2}=4 b^{2}|z(t)|^{2}$ and $\left|L_{t}\right|^{2}=4 \cos ^{2}(b s+\theta)\left|z^{\prime}(t)\right|^{2}$. Comparing these with (1.8) gives $|z|^{2}=1 /\left(4 b^{2}\right)$ and $\left|z^{\prime}\right|^{2}=1 / 4$. Therefore, the immersion is congruent to the immersion $L$ defined in statement (i).

Concerning statement (i) of Theorem 1 we provide the following theorem which insures the existence of the solution of the equation (1.1) subject to the two conditions. The proof uses the same idea as that of Theorem 4.1 in [3].

THEOREM 2. For any nonzero real number $b$ and any differentiable function $\theta$ of one variable defined on an open interval $I$, there exists a $\boldsymbol{C}^{2}$-valued solution of the differential equation:

$$
z^{\prime \prime}(t)-i \theta^{\prime}(t) z^{\prime}(t)+b^{2} z(t)=0,
$$

that also satisfies the two conditions: $|z|^{2}=1 /\left(4 b^{2}\right)$ and $\left|z^{\prime}\right|^{2}=1 / 4$.

Proof. Let $b$ be a nonzero number and $\theta(t)$ a differentiable function of one variable defined on an open interval $I$. Let $D$ be a simply-connected open domain in $\boldsymbol{R}^{2}=\{(s, t)$ : $s, t \in \boldsymbol{R}\}$ on which the function $\cos (b s+\theta(t))$ is nowhere zero.

Let $M=(D, g)$ denote the Riemannian 2-manifold equipped with metric tensor $g$ given by (1.8). Then its Levi-Civita connection satisfies (1.9). Let $\sigma$ denote the $T M$-valued symmetric bilinear form so that

$$
\begin{aligned}
& \sigma\left(\frac{\partial}{\partial s}, \frac{\partial}{\partial s}\right)=2 b \frac{\partial}{\partial s}, \quad \sigma\left(\frac{\partial}{\partial s}, \frac{\partial}{\partial t}\right)=b \frac{\partial}{\partial t}, \\
& \sigma\left(\frac{\partial}{\partial t}, \frac{\partial}{\partial t}\right)=b \cos ^{2}(b s+\theta(t)) \frac{\partial}{\partial s} .
\end{aligned}
$$


Then $\langle\sigma(X, Y), Z\rangle$ and $(\nabla \sigma)(X, Y, Z):=\nabla_{X} \sigma(U, Z)-\sigma\left(\nabla_{X} Y, Z\right)-\sigma\left(Y, \nabla_{X} Z\right)$ are totally symmetric. Moreover, a direct computation shows that the curvature tensor of $M$ satisfies $R(X, Y) Z=\sigma(\sigma(Y, Z), X)-\sigma(\sigma(X, Z), Y)$. Hence, by applying Theorem A in [1], we see that there exists a Lagrangian isometric immersion $\tilde{L}: M \rightarrow C^{2}$ whose second fundamental form $h=J \sigma$ satisfies (1.4). As in the proof of Theorem 1, we also see that the immersion satisfies (1.10).

After solving the first and the second equations of (1.10) in the same way as in the proof of Theorem 1, we obtain that

$$
\tilde{L}=e^{2 i b s} z(t)+\int_{0}^{t} z^{\prime}(t) e^{-2 i \theta(t)} d t+c
$$

for some $C^{2}$-valued function $z(t)$ and constant vector $c$. By substituting (1.19) into the last equation in (1.10), we discover

$$
z^{\prime \prime}(t)-i \theta^{\prime}(t) z^{\prime}(t)+b^{2} z(t)=0 .
$$

Thus, $z(t)$ is a solution of (1.17). Hence, by applying (1.8) and (1.19), we see that $z(t)$ satisfies the two side conditions: $|z|^{2}=1 /\left(4 b^{2}\right)$ and $\left|z^{\prime}\right|^{2}=1 / 4$ as well.

REMARK 1. Theorems 1 and 2 imply that the class of Lagrangian $H$-umbilical surfaces in $C^{2}$ satisfying (1.4) is very large.

REMARK 2. After the reparametrization $t=2 s$, the $C^{2}$-valued solution $z(s)$ given in Theorem 2 is nothing but a Legendre curve in the hypersphere $S^{3}(r) \subset C^{2}$ with radius $r=1 /(2 b)$.

REMARK 3. If $\theta=0$, the immersion defined by (1.2) is nothing but a Lagrangian pseudo-sphere as introduced in [1]. However, the immersion (1.2) is not a complex extensor in general.

EXAMPLE. Let $a, b$ be two real numbers with $8 a \neq b^{2}$ and $b \neq 0$. If $\theta=2 a t$, then the solution of (1.1) is given by

$$
z(t)=e^{i a t}\left(c_{1} e^{i \gamma t}+c_{2} e^{-i \gamma t}\right), \quad \gamma=\sqrt{a^{2}+b^{2}}
$$

for some constant vectors $c_{1}, c_{2} \in C^{2}$. Using (1.21) and the condition $|z|^{2}=1 /\left(4 b^{2}\right)$, we get

$$
\left|c_{1}\right|^{2}+\left|c_{2}\right|^{2}=\frac{1}{4 b^{2}}, \quad\left\langle c_{1}, c_{2}\right\rangle=\left\langle i c_{1}, c_{2}\right\rangle=0 .
$$

Moreover, from (1.21) and the condition $\left|z^{\prime}\right|^{2}=1 / 4$, we find

$$
(a+\gamma)^{2}\left|c_{1}\right|^{2}+(a-\gamma)^{2}\left|c_{2}\right|^{2}=\frac{1}{4} .
$$

Solving (1.22) and (1.23) for $\left|c_{1}\right|^{2}$ and $\left|c_{2}\right|^{2}$ yields

$$
\left|c_{1}\right|^{2}=\frac{\gamma-a}{8 b^{2} \gamma}, \quad\left|c_{2}\right|^{2}=\frac{\gamma+a}{8 b^{2} \gamma} .
$$


In view of (1.21) and (1.22), we may choose

$$
c_{1}=\left(\frac{\sqrt{\gamma-a}}{2 b \sqrt{2 \gamma}}, 0\right), \quad c_{2}=\left(0, \frac{\sqrt{\gamma+a}}{2 b \sqrt{2 \gamma}}\right) .
$$

Hence, the corresponding Lagrangian $H$-umbilical immersion is given by

$$
\begin{aligned}
& \tilde{L}=\frac{e^{i(\gamma-3 a) t}}{2 \sqrt{2} b \sqrt{\gamma}\left(b^{2}-8 a^{2}\right)}\left(\sqrt{\gamma-a}\left(4 a^{2}+b^{2}+4 a \gamma+\left(b^{2}-8 a^{2}\right) e^{2 i(b s+2 a t)}\right),\right. \\
& \left.\sqrt{\gamma+a}\left(4 a^{2}+b^{2}-4 a \gamma+\left(b^{2}-8 a^{2}\right) e^{2 i(b s+2 a t)}\right) e^{-2 i \gamma t}\right), \quad \gamma=\sqrt{a^{2}+b^{2}} .
\end{aligned}
$$

2. Lagrangian $H$-umbilical surfaces of constant curvature. Due to the error, we shall replace statement (ii-1) of Theorem 4.3 in [1] by the following result which completely classifies Lagrangian $H$-umbilical surfaces of constant curvature in $\boldsymbol{C}^{2}$.

THEOREM 3. Let $L: M \rightarrow C^{2}$ be a Lagrangian $H$-umbilical immersion of a surface of constant curvature $K$ into $C^{2}$. Then one of the following six statements holds:

(1) $K=0$ and $M$ is a Lagrangian cylinder over a curve.

(2) $M$ is a flat Lagrangian surface whose immersion is congruent to

$$
L(t, u)=u z(t)+\int_{0}^{t} b(t) z^{\prime}(t) d t
$$

where $b=b(t)$ is a real-valued function defined on an open interval $I \ni 0$ and $z: I \rightarrow$ $S^{3}(1) \subset C^{2}$ is a unit speed Legendre curve in $S^{3}(1)$.

(3) $K=b^{2}>0$ and $L$ is congruent to the Lagrangian immersion

$$
L(s, t)=e^{2 i b s} z(t)+\int_{0}^{t} z^{\prime}(t) e^{-2 i \theta(t)} d t,
$$

where $b$ is a positive number, $\theta$ is a function defined on an open interval $I \ni 0$ and $z: I \rightarrow$ $S^{3}(1 /(2 b)) \subset C^{2}$ is a Legendre curve with constant speed $1 / 2$ in $S^{3}(1 /(2 b))$ of radius $1 /(2 b)$ satisfying (1.1).

(4) $K=b^{2}>1$ and the immersion is congruent to

$$
\begin{aligned}
L(s, t)= & \frac{\cos (b s)}{\sqrt{b^{2}-1}} \exp \left(i \sin ^{-1}\left(\frac{b \sin b s}{\sqrt{b^{2}-1}}\right)-\frac{i}{b} \tan ^{-1}\left(\frac{\sin b s}{\sqrt{b^{2} \cos ^{2} b s-1}}\right)\right) \\
& \times\left(i c_{1}+\cos \left(\sqrt{b^{2}-1} t\right), i c_{2}+\sin \left(\sqrt{b^{2}-1} t\right)\right) \\
& +\left(\int _ { 0 } ^ { s } \operatorname { e x p } \left\{2 i \sin ^{-1}\left(\frac{b \sin b s}{\sqrt{b^{2}-1}}\right)\right.\right. \\
& \left.\left.-\frac{i}{b} \tan ^{-1}\left(\frac{\sin b s}{\sqrt{b^{2} \cos ^{2} b s-1}}\right)\right\} d s\right)\left(c_{1}, c_{2}\right)
\end{aligned}
$$

for some constants $c_{1}, c_{2}$. 
(5) $K=b^{2}>0$ and the immersion is congruent to

$$
\begin{aligned}
L(s, t)= & \frac{\cos (b s)}{\sqrt{b^{2}+1}} \exp \left(i \sin ^{-1}\left(\frac{b \sin b s}{\sqrt{b^{2}+1}}\right)+\frac{i}{b} \tanh ^{-1}\left(\frac{\sin b s}{\sqrt{b^{2} \cos ^{2} b s+1}}\right)\right) \\
& \times\left(i c_{1}+\cos \left(\sqrt{b^{2}+1} t\right), i c_{2}+\sin \left(\sqrt{b^{2}+1} t\right)\right) \\
& +\left(\int _ { 0 } ^ { s } \operatorname { e x p } \left\{2 i \sin ^{-1}\left(\frac{b \sin b s}{\sqrt{b^{2}+1}}\right)\right.\right. \\
& \left.\left.+\frac{i}{b} \tanh ^{-1}\left(\frac{\sin b s}{\sqrt{b^{2} \cos ^{2} b s+1}}\right)\right\} d s\right)\left(c_{1}, c_{2}\right)
\end{aligned}
$$

for some constants $c_{1}, c_{2}$.

(6) $K=-b^{2}<0$ and the immersion is congruent to

$$
\begin{aligned}
L(s, t)= & \frac{\cosh (b s)}{\sqrt{1-b^{2}}} \exp \left(i \sin ^{-1}\left(\frac{b \sinh b s}{\sqrt{1-b^{2}}}\right)+\frac{i}{b} \tan ^{-1}\left(\frac{\sinh b s}{\sqrt{1-b^{2} \cosh ^{2} b s}}\right)\right) \\
& \times\left(i c_{1}+\cos \left(\sqrt{1-b^{2}} t\right), i c_{2}+\sin \left(\sqrt{1-b^{2}} t\right)\right) \\
& +\left(\int _ { 0 } ^ { s } \operatorname { e x p } \left\{2 i \sin ^{-1}\left(\frac{b \sinh b s}{\sqrt{1-b^{2}}}\right)\right.\right. \\
& \left.\left.+\frac{i}{b} \tan ^{-1}\left(\frac{\sinh (b s)}{\sqrt{1-b^{2} \cosh ^{2}(b s)}}\right)\right\} d s\right)\left(c_{1}, c_{2}\right)
\end{aligned}
$$

for some constants $c_{1}, c_{2}$.

Proof. Let $L: M \rightarrow C^{2}$ be a Lagrangian $H$-umbilical immersion of a surface of constant curvature $K$ into $C^{2}$. Then, by definition, the second fundamental form of $L$ satisfies

$$
h\left(e_{1}, e_{1}\right)=\lambda J e_{1}, \quad h\left(e_{1}, e_{2}\right)=\mu J e_{2}, \quad h\left(e_{2}, e_{2}\right)=\mu J e_{1}
$$

for some functions $\lambda, \mu$ with respect to some orthonormal frame field $e_{1}, e_{2}$. From (2.6) and the equation of Codazzi, we find

$$
\begin{aligned}
& e_{1} \mu=(\lambda-2 \mu) \omega_{1}^{2}\left(e_{2}\right), \quad e_{2} \mu=3 \mu \omega_{1}^{2}\left(e_{1}\right), \\
& e_{2} \lambda=(\lambda-2 \mu) \omega_{1}^{2}\left(e_{1}\right),
\end{aligned}
$$

where $\nabla_{X} e_{1}=\omega_{1}^{2}(X) e_{2}$. Using (2.6) and the equation of Gauss, we find

$$
\lambda \mu-\mu^{2}=K=\text { constant }
$$

Differentiating (2.8) with respect to $e_{2}$ and applying (2.7) yield $\mu(\lambda-2 \mu) \omega_{2}^{1}\left(e_{1}\right)=0$. Thus, we have either $\mu(\lambda-2 \mu)=0$ or $\omega_{1}^{2}\left(e_{1}\right)=0$.

If $\mu(\lambda-2 \mu)=0$, then we obtain $K=0$ or $\lambda=2 \mu$. Thus, by applying the Main Theorem of [2] and Theorem 1, we have statements (1), (2) or (3).

If $\mu(\lambda-2 \mu) \neq 0$, we get $\omega_{1}^{2}\left(e_{1}\right)=0$. So, from (2.7), we find that $e_{2} \lambda=e_{2} \mu=0$. Since $\nabla_{e_{1}} e_{1}=0$, there is a local coordinate system $\{s, u\}$ on $M$ such that the metric tensor is 
given by

$$
g=d s^{2}+G^{2}(s, u) d u^{2}
$$

for some function $G$ with $\partial / \partial s=e_{1}, \partial / \partial u=G e_{2}$. From $e_{2} \lambda=e_{2} \mu=0$, we get $\lambda=\lambda(s)$ and $\mu=\mu(s)$. Using (2.9), we find

$$
\nabla_{\partial / \partial u} \frac{\partial}{\partial s}=(\ln G)_{s} \frac{\partial}{\partial u}, \quad \omega_{1}^{2}\left(e_{2}\right)=\frac{G_{s}}{G} .
$$

Now, let us assume that $\lambda \neq 2 \mu$. Then, by (2.7)-(2.10), we obtain

$$
(\ln G)_{s}=\frac{\mu^{\prime}}{\lambda-2 \mu}=\frac{\mu \mu^{\prime}}{K-\mu^{2}} .
$$

Solving this equation gives $G=F(u) / \sqrt{\left|K-\mu^{2}\right|}$ for some function $F$. Thus, (2.9) becomes

$$
g=d s^{2}+\frac{F^{2}(u)}{\left|K-\mu^{2}(s)\right|} d u^{2} .
$$

If $t$ denotes an antiderivative of $F(u)$, then we obtain from (2.11) that

$$
g=d s^{2}+\frac{d t^{2}}{\left|K-\mu^{2}(s)\right|}, \quad G^{2}(s)=\frac{1}{\left|K-\mu^{2}(s)\right|} .
$$

CASE (a) $K=b^{2}>0$. Since $G$ satisfies $G_{s s}+b^{2} G=0$ in this case, we have $G=c_{1} \cos (b s)+c_{2} \sin (b s)$ for some constants $c_{1}, c_{2}$, not both zero. Thus, we obtain $G=$ $r \cos (b s+c)$ for some constants $r \neq 0$ and $c$. After applying a suitable translation in $s$ and a suitable dilation in $t$, we obtain $G(s)=\cos (b s)$. Therefore, we find

$$
\begin{aligned}
& g=d s^{2}+\cos ^{2}(b s) d t^{2} \\
& \nabla_{\partial / \partial s} \frac{\partial}{\partial s}=0, \quad \nabla_{\partial / \partial s} \frac{\partial}{\partial t}=-b \tan (b s) \frac{\partial}{\partial t} \\
& \nabla_{\partial / \partial t} \frac{\partial}{\partial t}=\frac{b}{2} \sin (2 b s) \frac{\partial}{\partial s} .
\end{aligned}
$$

CASE (a-1) $K=b^{2}>\mu^{2}$ and $b>0 . \quad$ In this case, we have $b^{2}=\mu^{2}+\sec ^{2}(b s) \geq 1$. Without loss of generality, we may assume that

$$
\lambda=\frac{2 b^{2}-\sec ^{2}(b s)}{\sqrt{b^{2}-\sec ^{2}(b s)}}, \quad \mu=\sqrt{b^{2}-\sec ^{2}(b s)} .
$$

By applying (2.6), (2.13) and (2.14), we see that the immersion satisfies

$$
\begin{aligned}
& L_{s s}=i \frac{2 b^{2}-\sec ^{2}(b s)}{\sqrt{b^{2}-\sec ^{2}(b s)}} L_{s}, \\
& L_{s t}=\left(i \sqrt{b^{2}-\sec ^{2}(b s)}-b \tan (b s)\right) L_{t}, \\
& L_{t t}=\left(i \sqrt{b^{2} \cos ^{2}(b s)-1}+b \sin (b s)\right) \cos (b s) L_{s} .
\end{aligned}
$$


After solving the second equation of (2.15), we get

$$
L_{t}=F(t) \cos (b s) \exp \left(i \sin ^{-1}\left(\frac{b \sin b s}{\sqrt{b^{2}-1}}\right)-\frac{i}{b} \tan ^{-1}\left(\frac{\sin b s}{\sqrt{b^{2} \cos ^{2} b s-1}}\right)\right)
$$

for some $\boldsymbol{C}^{2}$-valued function $F(t)$. Thus, we have

$$
\begin{aligned}
L= & A(s)+B(t) \cos (b s) \exp \left(i \sin ^{-1}\left(\frac{b \sin b s}{\sqrt{b^{2}-1}}\right)\right. \\
& \left.-\frac{i}{b} \tan ^{-1}\left(\frac{\sin b s}{\sqrt{b^{2} \cos ^{2} b s-1}}\right)\right),
\end{aligned}
$$

where $B(t)$ is an antiderivative of $F(t)$ and $A(s)$ is a $C^{2}$-valued function. From (2.17) we find

$$
\begin{aligned}
L_{s}= & A^{\prime}(s)-B(t)\left(b \sin b s-i \sqrt{b^{2} \cos ^{2} b s-1}\right) \\
& \times \exp \left(i \sin ^{-1}\left(\frac{b \sin b s}{\sqrt{b^{2}-1}}\right)-\frac{i}{b} \tan ^{-1}\left(\frac{\sin b s}{\sqrt{b^{2} \cos ^{2} b s-1}}\right)\right), \\
L_{s s}= & A^{\prime \prime}(s)-B(t) \frac{\left(\sqrt{b^{2}-\sec ^{2} b s}+i b \tan b s\right)\left(2 b^{2} \cos ^{2} b s-1\right)}{\sqrt{b^{2} \cos ^{2} b s-1}} \\
& \times \exp \left(i \sin ^{-1}\left(\frac{b \sin b s}{\sqrt{b^{2}-1}}\right)-\frac{i}{b} \tan ^{-1}\left(\frac{\sin b s}{\sqrt{b^{2} \cos ^{2} b s-1}}\right)\right) .
\end{aligned}
$$

Substituting (2.18) and (2.19) into the first equation in (2.15), we find

$$
A^{\prime \prime}(s)=i \frac{2 b^{2}-\sec ^{2}(b s)}{\sqrt{b^{2}-\sec ^{2}(b s)}} A^{\prime}(s) .
$$

Solving this equation gives

$$
A^{\prime}(s)=C \exp \left\{2 i \sin ^{-1}\left(\frac{b \sin b s}{\sqrt{b^{2}-1}}\right)-\frac{i}{b} \tan ^{-1}\left(\frac{\sin b s}{\sqrt{b^{2} \cos ^{2} b s-1}}\right)\right\}
$$

for some vector $C$ in $C^{2}$. Hence, we obtain

$$
A(s)=C \int_{0}^{s} \exp \left\{2 i \sin ^{-1}\left(\frac{b \sin b s}{\sqrt{b^{2}-1}}\right)-\frac{i}{b} \tan ^{-1}\left(\frac{\sin b s}{\sqrt{b^{2} \cos ^{2} b s-1}}\right)\right\} d s+E
$$

for vector $E$ in $\boldsymbol{C}^{2}$. We may assume $E=0$ by applying a suitable translation on $\boldsymbol{C}^{2}$. Hence, we obtain from (2.17) that

$$
\begin{aligned}
L= & C \int_{0}^{s} \exp \left\{2 i \sin ^{-1}\left(\frac{b \sin b s}{\sqrt{b^{2}-1}}\right)-\frac{i}{b} \tan ^{-1}\left(\frac{\sin b s}{\sqrt{b^{2} \cos ^{2} b s-1}}\right)\right\} d s \\
& +B(t) \cos (b s) \exp \left(i \sin ^{-1}\left(\frac{b \sin b s}{\sqrt{b^{2}-1}}\right)-\frac{i}{b} \tan ^{-1}\left(\frac{\sin b s}{\sqrt{b^{2} \cos ^{2} b s-1}}\right)\right) .
\end{aligned}
$$


Thus we have

$$
\begin{aligned}
L_{s}= & \left(C+i \sqrt{b^{2}-1} B\right) \exp \left(2 i \sin ^{-1}\left(\frac{b \sin b s}{\sqrt{b^{2}-1}}\right)\right. \\
& \left.-\frac{i}{b} \tan ^{-1}\left(\frac{\sin b s}{\sqrt{b^{2} \cos ^{2} b s-1}}\right)\right),
\end{aligned}
$$

$$
L_{t}=B^{\prime}(t) \cos (b s) \exp \left(i \sin ^{-1}\left(\frac{b \sin b s}{\sqrt{b^{2}-1}}\right)-\frac{i}{b} \tan ^{-1}\left(\frac{\sin b s}{\sqrt{b^{2} \cos ^{2} b s-1}}\right)\right) .
$$

Substituting (2.24) and (2.25) into the last equation of (2.15) yields

$$
B^{\prime \prime}(t)+\left(b^{2}-1\right) B(t)=i \sqrt{b^{2}-1} C,
$$

which implies that

$$
B(t)=C_{1} \cos \sqrt{b^{2}-1} t+C_{2} \sin \sqrt{b^{2}-1} t+\frac{i}{\sqrt{b^{2}-1}} C .
$$

Substituting these into (2.23) gives

$$
\begin{aligned}
L= & C \int_{0}^{s} \exp \left\{2 i \sin ^{-1}\left(\frac{b \sin b s}{\sqrt{b^{2}-1}}\right)-\frac{i}{b} \tan ^{-1}\left(\frac{\sin b s}{\sqrt{b^{2} \cos ^{2} b s-1}}\right)\right\} d s \\
& +\left(C_{1} \cos \sqrt{b^{2}-1} t+C_{2} \sin \sqrt{b^{2}-1} t+\frac{i}{\sqrt{b^{2}-1}} C\right) \cos (b s) \\
& \times \exp \left(i \sin ^{-1}\left(\frac{b \sin b s}{\sqrt{b^{2}-1}}\right)-\frac{i}{b} \tan ^{-1}\left(\frac{\sin b s}{\sqrt{b^{2} \cos ^{2} b s-1}}\right)\right) .
\end{aligned}
$$

Hence, we may obtain (2.3) by choosing suitable initial conditions.

CASE (a-2) $K=b^{2}<\mu^{2}$ and $b>0$. In this case, we have $\mu^{2}-b^{2}=\sec ^{2}(b s)$. Hence, without loss of generality, we may assume that

$$
\lambda=\frac{2 b^{2}+\sec ^{2}(b s)}{\sqrt{b^{2}+\sec ^{2}(b s)}}, \quad \mu=\sqrt{b^{2}+\sec ^{2}(b s)} .
$$

By applying (2.6), (2.13) and (2.28), we obtain

$$
\begin{aligned}
& L_{s s}=i \frac{2 b^{2}+\sec ^{2}(b s)}{\sqrt{b^{2}+\sec ^{2}(b s)}} L_{s}, \\
& L_{s t}=\left(i \sqrt{b^{2}+\sec ^{2}(b s)}-b \tan (b s)\right) L_{t}, \\
& L_{t t}=\left(i \sqrt{b^{2} \cos ^{2}(b s)+1}+b \sin (b s)\right) \cos (b s) L_{s} .
\end{aligned}
$$

After solving this system of PDEs in the same way as in Case (a-1) and after choosing suitable initial conditions, we conclude that the immersion is congruent to (2.4).

CASE (b) $K=-b^{2}<0$ and $b>0$. Since $G$ satisfies $G_{s s}-b^{2} G=0$ in this case, we get $G=c_{1} \cosh (b s)+c_{2} \sinh (b s)$ for some constants $c_{1}, c_{2}$, not both zero. Thus, by applying 
a suitable translation in $s$ and a suitable dilation in $t$, we obtain

$$
\begin{aligned}
& g=d s^{2}+\cosh ^{2}(b s) d t^{2}, \\
& \nabla_{\partial / \partial s} \frac{\partial}{\partial s}=0, \quad \nabla_{\partial / \partial s} \frac{\partial}{\partial t}=b \tanh (b s) \frac{\partial}{\partial t}, \\
& \nabla_{\partial / \partial t} \frac{\partial}{\partial t}=-\frac{b}{2} \sinh (2 b s) \frac{\partial}{\partial s} .
\end{aligned}
$$

From (2.12) and the first equation in (2.30), we find $\operatorname{sech}^{2} b s=b^{2}+\mu^{2}$. Thus we obtain $1 \geq \operatorname{sech}^{2} b s \geq b^{2}$ and $\mu= \pm \sqrt{\operatorname{sech}^{2} b s-b^{2}}$. Without loss of generality, we may assume that

$$
\lambda=\frac{\operatorname{sech}^{2}(b s)-2 b^{2}}{\sqrt{\operatorname{sech}^{2}(b s)-b^{2}}}, \quad \mu=\sqrt{\operatorname{sech}^{2}(b s)-b^{2}} .
$$

By applying (2.6), (2.30) and (2.31), we see that the immersion satisfies

$$
\begin{aligned}
& L_{s s}=i \frac{\operatorname{sech}^{2}(b s)-2 b^{2}}{\sqrt{\operatorname{sech}^{2}(b s)-b^{2}}} L_{s}, \\
& L_{s t}=\left(i \sqrt{\operatorname{sech}^{2}(b s)-b^{2}}+b \tanh (b s)\right) L_{t}, \\
& L_{t t}=\left(i \sqrt{1-b^{2} \cosh ^{2}(b s)}-b \sinh (b s)\right) \cosh (b s) L_{s} .
\end{aligned}
$$

After solving this system of PDEs in the same way as in Case (a-1) and after choosing suitable initial conditions, we conclude that the immersion $L$ is congruent to (2.5).

\section{REFERENCES}

[1] B.-Y. CHEN, Complex extensors and Lagrangian submanifolds in complex Euclidean spaces, Tôhoku Math. J. 49 (1997), 277-297.

[2] B.-Y. CHEN, Representation of flat Lagrangian $H$-umbilical submanifolds in complex Euclidean spaces, Tohoku Math. J. 51 (1999), 13-20.

[ 3 ] B.-Y. CHEN, Classification of flat slant surfaces in complex Euclidean plane, J. Math. Soc. Japan 54 (2002), 719-746.

DEPARTMENT OF MATHEMATICS

Michigan STATE UnIVERSITY

EAST LANSING, MI 48824-1027

U.S.A.

E-mail address: bychen@math.msu.edu 